\title{
The Relation of the Electric Threshold and Accommodation in the Human Retina to Brightness Discrimination
}

\author{
By \\ Makoto Sato \\ (佐 藤 誠) \\ From the Department of Physiology, Iwate Medical College, \\ Morioka; Director: Prof. T. Mita
}

(Received for publication, September 13, 1958)

\section{INTRODUCTION}

The author investigated in a previous paper ${ }^{11}$ the relation between the electric threshold (V) and the radiance (I) of the adapting colored light, and reported that there were two different types in the form of the V-log I curve depending upon the different time-constant (RC) of the exponentially increasing current, i.e., one was a group of the curves for $\mathrm{RC}$ shorter than $25 \mathrm{msec}$, and the other was a group of those for RG longer than $75 \mathrm{msec}$. For mathematical expression of these V-log I curves, he had assumed the following equation for the relation between the electric threshold (V) and the intensity (I) of the adapting light,

$$
\mathrm{V}=\mathrm{A} \log \Delta \mathrm{I}=\mathrm{A}[\log (\Delta \mathrm{I} / \mathrm{I})+\log \mathrm{I}] \ldots \ldots \ldots \ldots \ldots \ldots(1),
$$

where $\mathrm{A}$ is a proportionality constant and $\Delta \mathrm{I}$ is the least perceptible brightness increment to be added to the brightness (I) of the background, so that $\Delta \mathrm{I} / \mathrm{I}$ means the Weber fraction (W). Using the data of Steinhardt and Blackwell's experiments ${ }^{23)}$ concerning the Weber fraction, we obtained $\mathrm{V}$ by equation (1). The thus calculated V-log I curve was well consistent in shape with the curves obtained experimentally by the currents of RC longer than $75 \mathrm{msec}$., however it did not agree with those obtained by the currents of RC shorter than $25 \mathrm{msec}$.

In this paper, the author attempted to get a mathematical formula applicable not only to the former case but also to the latter case. And, in order to confirm the validity of such an hypothetical formula, the relation between the accommodation constant (Hill's $\lambda^{4}$ ) and the intensity of the adapting light was investigated precisely with the eyes adapted to monochromatic lights of varying wavelengths. 


\section{EXPERIMENTAL \\ Methods}

As a stimulating electrode, a silver plate of $2 \times 3 \mathrm{~cm}$. in size was inserted between the upper lip and gum, while another large silver plate was placed under the right sole, being immersed into $1 \%$ saline as an indifferent electrode. Taking the electrical phosphene as an index of excitation, the electric thresholds (V) were determined for the varying time-constants $(\mathrm{RC})$ of the stimulating current. Measurements of $\mathrm{V}$ were made by the same procedure as reported in the previous paper ${ }^{5}$. Every adapting colored light of different wavelengths was isolated by a set of metal interference filters of Lange \& Co., whose spectral transmittance must be referred to Yaegashi's paper ${ }^{6}$. The radiance (I) of the surface emitting the adapting light was measured by a system of a thermopile and a galvanometer.

\section{Results}

a) Dependence of the form of the accommodation curve on the wavelength and on the radiance of the adapting light

In order to look over the dependence of the accommodation curve on the wavelength of the adapting light, three monochromatic rays of 430, 550 and $700 \mathrm{~m} \mu$ in wavelength were first employed as the adapting light. In these cases, these monochromatic lights used were of an equal energy level high enough for photopic vision. The curves obtained are shown in Fig. 1 (A).

As can be seen in Fig. 1 (A), the slope of the curve becomes steeper in the order of 700,430 and $550 \mathrm{~m} \mu$. This fact suggests that the change in slope of the curves is due to the spectral luminosity of the adapting lights. The curves for 430 and $700 \mathrm{~m} \mu$ show an abrupt inflexion at about 50-80

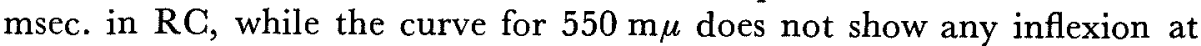
this range of $\mathrm{RC}$. All these findings are well in conformity with the results which were obtained by Sato, Suzuki and Akihama ${ }^{5)}$ with the eye adapted to various intensity levels of white light. It was found that the form of the accommodation curve depends on the intensity of the adapting light, and that an intensity level to produce a straight form of the accommodation curve without inflexion, is approximately $1.2 \times 10$ lux. Accordingly, the radiance of the adapting light of $550 \mathrm{~m} \mu$ used in this experiment would give the same illuminance as $1.2 \times 10$ lux of white light.

Secondly, the dependence of the form of the accommodation curve on the radiance of the adapting light was investigated for varying wavelengths at 430, 500, 550, 670 and $700 \mathrm{~m} \mu$, and some data of the measurements were plotted in Fig. 1 (B), (C) and (D), others were shown in Table I. 

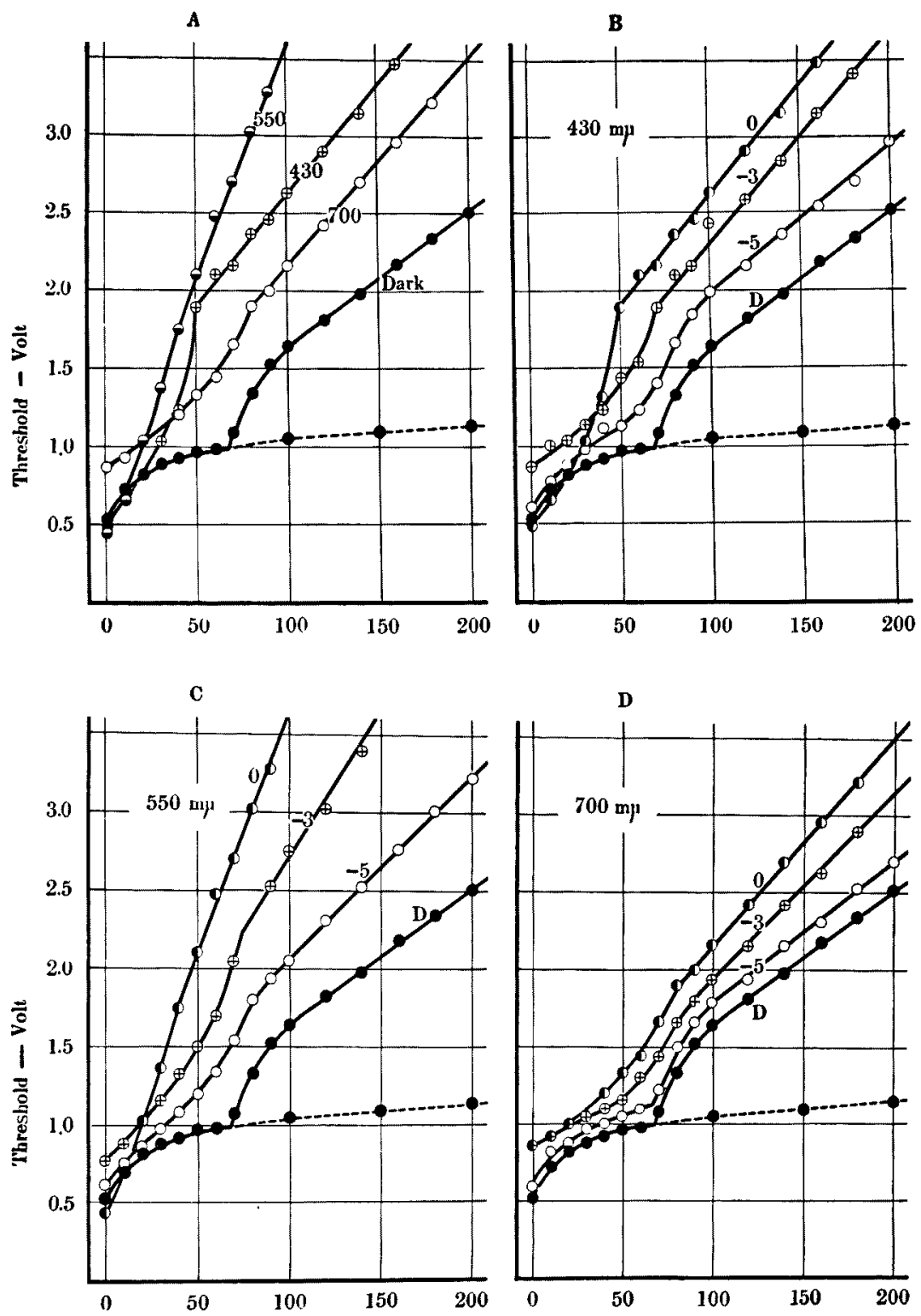

$\mathrm{RC}$ - $\mathrm{msec}$

RC - msec

Fig. 1. (A) shows the accommodation curves for three different wavelengths of the adapting light whose radiances were fixed at an equal energy enough for photopic vision. Numbers on the curves are wavelength in $\mathrm{m} \mu$. (B), (C) and (D) show the dependence of the accommodation curve on the radiance of the adapting light of 430,550 and $700 \mathrm{~m} \mu$ in wavelength respectively. Filled circles are data obtained from the dark adapted eyes. 


\section{TAB LE I}

Dependence of the Electric Threshold on the Time-constant and on the Radiance of the Adapting Light

Electric thresholds are shown in volt. Subject: M. Sato (Exp. No. $560120)$,

\begin{tabular}{|c|c|c|c|c|c|c|c|}
\hline \multirow{2}{*}{$\begin{array}{l}\text { Radiance } \rightarrow \\
\text { Time const. } \\
\text { in msec. } \downarrow\end{array}$} & \multicolumn{4}{|c|}{$(500 \mathrm{~m} \mu)$} & \multicolumn{3}{|c|}{$(670 \mathrm{~m} \mu)$} \\
\hline & Dark & $10^{-5}$ & $10^{-8}$ & 1 & $10^{-5}$ & $10^{-8}$ & 1 \\
\hline 0 & 0.52 & 0.80 & 0.75 & 0.54 & 0.62 & 0.88 & 0.78 \\
\hline 10 & 0.68 & 0.95 & 0.82 & 0.82 & 0.80 & 0.95 & 0.85 \\
\hline 20 & 0.80 & 1.03 & 0.90 & 1.08 & 0.85 & 1.05 & 1.03 \\
\hline 30 & 0.88 & 1.11 & 1.00 & 1.33 & 0.95 & 1.10 & 1.10 \\
\hline 40 & 0.98 & 1.22 & 1.13 & 1.58 & 0.99 & 1.19 & 1.26 \\
\hline 50 & 0.96 & 1.38 & 1.38 & 1.85 & 1.05 & 1.30 & 1.44 \\
\hline 60 & 0.98 & 1.58 & 1.79 & 2.10 & 1.10 & 1.37 & 1.91 \\
\hline 70 & 1.06 & 1.80 & 2.05 & 2.36 & 1.30 & 1.54 & 1.92 \\
\hline 80 & 1.32 & 2.10 & 2.26 & 2.63 & 1.52 & 1.74 & 2.00 \\
\hline 90 & 1.50 & 2.26 & 2.42 & 2.90 & 1.66 & 1.90 & 2.26 \\
\hline 100 & 1.64 & 2.42 & 2.54 & 3.15 & 1.80 & 2.00 & 2.36 \\
\hline 120 & 1.82 & 2.63 & 2.90 & 3.60 & 1.99 & 2.26 & 2.63 \\
\hline 140 & 1.98 & 2.96 & 3.20 & & 2.16 & 2.53 & 2.96 \\
\hline 160 & 2.16 & 3.15 & 3.59 & & 2.36 & 2.76 & 3.28 \\
\hline 180 & 2.34 & 3.42 & & & 2.48 & 3.02 & 3.47 \\
\hline 200 & 2.52 & & & & 2.70 & 3.28 & \\
\hline
\end{tabular}

As can be seen in Fig. 1 (B), (C) and (D), the slope of the curves becomes steeper as the radiance is raised progressively. The rate of variation in slope with increase in radiance of the adapting light is also dependent upon the wavelength, that is to say: this rate increases in the order of 700,430 and $550 \mathrm{~m} \mu$.

b) Relation between Hill's $\lambda$ and log-radiance $(I)$ of the adapting light

As shown in Fig. 1, most accommodation curves show an inflexion at about $50-80$ msec. in RC. The curve is divided by this inflexion into a "left section" and a "right section" for time-constants shorter and longer than this critical value of RC, respectively. Now, from both sections of an accommodation curve, two values of Hill's $\lambda$ were obtained separately. The data obtained from the "left section" were plotted in Fig. 2 (A) and those obtained from the "right section" in Fig. 2 (B).

The value of $\lambda$ in Fig. 2 (A) decreases rapidly showing an upward concave curve with increase in radiance from dark to $10^{-3}$ unit (relative value). Then, it decreases again, marking a faint inflexion when the radi- 


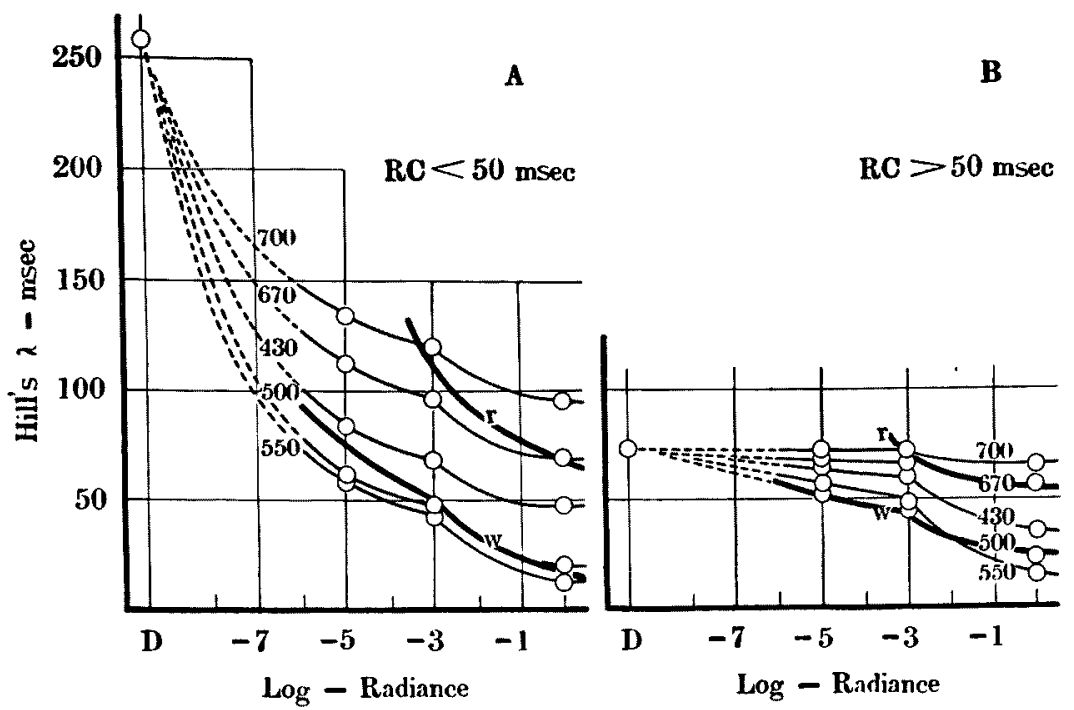

Fig. 2. The relation between Hill's $\lambda$ and log-radiance of the adapting light. (A) and (B) are cases for $\mathrm{RC}<50 \mathrm{msec}$. and $\mathrm{RC}>50 \mathrm{msec}$. respectively. Numbers on the curves are wavelength of the adapting light in $m \mu$. $D$ means complete dark. The thick curves denoted by $\mathbf{r}$ and $w$ show the $\lambda$-Iog I relation calculated from the equation $\left(5^{\prime}\right)$ by using Steinhardt's data for the red and white light respectively.

ance is raised further. On the other hand, the value of $\lambda$ in Fig. 2 (B) decreases gradually with a gentle slope till the radiance arrives at $10^{-3}$ unit, and then decreases rapidly, showing a clear break with further increase in radiance. Thus, there was found a remarkable difference in form between both kinds of the $\lambda$-log $I$ curves obtained from the left and right sections. This result seems to indicate a different seat of excitation in the retina, depending on whether the stimulating current of $\mathrm{RC}$ is longer or shorter than 50 msec., as inferred by Mita et al. ${ }^{7)}$.

5 curves of $\lambda-\log I$ relation in Fig. 2 (A) or (B) show the systematic vertical displacement according to the wavelength of the adapting light. As can be scen in Fig. 2 (B), the magnitudes of these vertical displacements are proportional to the spectral luminosity of the adapting monochromatic lights respectively. Attention should be directed to the fact that the order of this vertical shifting differs depending upon whether the intensity range of the adapting light is scotopic or photopic. That is, within the scotopic range, the curves for varying wavelengths shift downwards in a parallel relation to the scotopic luminosity of the spectrum, while in the photopic range, the curves to the photopic luminosity of the spectrum. It is further to be noted that the hue of the adapting light can be perceived at first at 
the radiance corresponding to this break on the curve. The curves for the red end of the spectrum show little variation within a scotopic range of the radiance. This is obviously due to the low stimulating efficiency of red light for rod. From these facts, it can be deduced that the inflexion on the $\lambda-\log I$ curves at about $10^{-3}$ unit in radiance is caused by the independent function of the two types of receptors, namely rods and cones, and it was assumed that the neurons stimulated by the currents of $\mathrm{RC}$ longer than $50 \mathrm{msec}$. may be closely related to the primary receptive neurons. On the other hand, in Fig. 2 (A), the order of the downward shifting of the curves corresponds always to the photopic luminosity of the spectrum, irrespective of whether the adaptaion was scotopic or photopic. Moreover, the curves for the red end of the spectrum show a far steeper slope than in the former case (Fig. 2 (B)) even within a scotopic range in radiance. In addition, the inflexion of the $\lambda$-log $I$ curve at about $10^{-3}$ unit does not appear so clearly as in the case of Fig. 2 (B). From these facts, it seems likely that the neural elements stimulated by the current of RC shorter than 50 msec. would not be so closely related to the receptor neurons as in the former case, but probably to some other secondary neurons, such as the bipolar cells and horizontal cells.

\section{Discussion}

a) Dependence of the electric threshold $(V)$ on the radiance $(I)$ of the adapting colored light and on the time-constant $(R C)$ of the stimulating current

As the author reported in the previous paper ${ }^{11}$, equation (1) was applicable only to the case of RC longer than $75 \mathrm{msec}$, but it was not applicable to the case of RC shorter than $25 \mathrm{msec}$. In order to get a mathematical formula applicable not only to the former case but also to the latter, the author introduced a parameter $p$ into equation (1), as follows :

$$
\mathrm{V}=\mathrm{A}\left(\log \mathrm{W}^{\mathrm{p}}+\log \mathrm{I}\right)+\mathrm{B}
$$

where B is a constant independent of I and W. Equation (1) can be regarded as the special case for $p=1$ in equation (2). Now, using Steinhardt's data ${ }^{2)}$ for $I$ and $W$, and putting $p=1,1.5,2,3,3.5$, we get five curves from equation (2) as shown in Fig. 3, where $A=3.1^{-1}, B=3.7-0.71 \mathrm{p}$ in the cases of $p \leqq 2$ and $B=3.7-0.55 p$ in the cases of $p \geqq 3$. As can be seen in Fig. 3, each curve for $\mathrm{p}=1,1.5,2,3,3.5$ agrees approximately with the experimental data (shown by circles) for time-constant $(\mathrm{RG})=100$, $75,50,25,0$ msec. respectively. Furthermore, if we compare the above calculated curve with the V-log I curves which were reported by Mita, Fujimaki \& Takahashi" ${ }^{7)}$ and Mita, Fujimaki \& Yaegashi ${ }^{8)}$, in the case of stimulation with the sinusoidal alternating current, it is found that the 


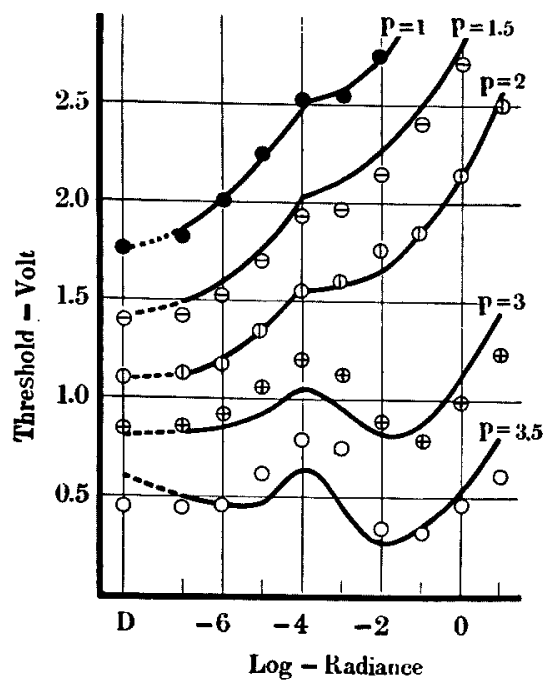

Fig. 3. Curves show the V-log I relation calculated from equation (2) by using the Steinhardt's data and by putting $p=1,1.5,2,3,3.5$. Circles show the experimental data ubtained with the eye adapted to monochromatic light of $550 \mathrm{~m} \mu$ in wavelength. The time-constants of the stimulating currents used are as follows, $: 100 \mathrm{msec} ., \Theta: 75 \mathrm{msec}$, (1) : $50 \mathrm{msec}$, $\oplus: 25 \mathrm{msec}$., $\bigcirc: 0$ msec.

$\mathrm{V}-\log \mathrm{I}$ curves for $\mathrm{p}=1,3,5$ in equation (2) are quite similar to those for frequencies at 3,30 and $1000 \mathrm{cps}$. respectively. These facts demonstrate that the parameter $p$ in equation (2) can be regarded as a function of the experimental condition of electrostimulation, such as time-constant (RC) and frequency of the stimulating currents. It is further to be noted that in the cases of electrostimulation of the eye with A. C. also, V-log I curves can be represented satisfactorily in a single equation (2) by taking some suitable value of $p$.

Equation (2) can be expressed also in another form,

$$
\mathrm{V}=\mathrm{A} \log \Delta \mathrm{I}(\Delta \mathrm{I} / \mathrm{I})^{\mathrm{p}-1}+\mathrm{B}
$$

In the case for $p=1$, equation (3) means that the electric threshold is simply proportional to the logarithm of the least perceptible brightness increment $(\Delta \mathrm{I})$, just as if the structures stimulated were the primary receptive neurons. On the other hand, in the cases for $\mathrm{p}=2$ or more, the equation (3) expresses that the electric threshold is not simply proportional to the $\log$ I, so that it should be determined by another more complicated mechanism of excitation than in the case for $p=1$. These facts also afford evidence supporting the Mita et al.'s working hypothesis with regard to the different seat of stimulation in the retina. 
b) Dependence of the Hill's $\lambda$ upon the intensity of the adapting light

The fact that Hill's accommodation constant $\lambda$ of the human retina for electrostimulation varies with varying intensities of the adapting light, was first reported by Motokawa \& Iwama9). Now, a question arises why $\lambda$ varies depending on the adaptation levels, and what mechanism causes this phenomenon. In this connection, the author attempted to answer the problem from the viewpoint of the brightness discrimination, as stated above. As the parameter $\mathrm{p}$ is considered to be a function of the timeconstant (RC) of the stimulating current, we get the following formula, by differentiating the equation (2) with respect to $\mathrm{RC}$,

$$
\partial \mathrm{V} / \partial \mathrm{t}=\mathrm{A}(\log \mathrm{W}) \partial \mathrm{p} / \partial \mathrm{t}
$$

where $\mathrm{t}$ denotes the RC. According to Hill's theory, the accommodation constant $\lambda$ is presented by the reciprocal of the tangent of the V-RC curve when $\lambda / \mathrm{k}$ is large and $\mathrm{RC}$ of the stimulating current is far larger than $\mathrm{k}$ and $\lambda$. Then, we obtain,

$$
\begin{aligned}
& \partial \mathrm{V} / \partial \mathrm{t}=\mathrm{A}(\log \mathrm{W}) \partial \mathrm{p} / \partial \mathrm{t}=\mathrm{V}_{\mathrm{o}} / \lambda \ldots \ldots \ldots \ldots \ldots \ldots \ldots \ldots \ldots,(5),
\end{aligned}
$$

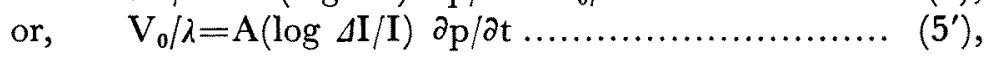

where $V_{0}$ means the rheobase. $V_{0}, \lambda$ and $A$ in the equation (5) were already known by this experiment and $\log W$ is referable to Steinhardt's $\mathrm{data}^{2)}$. Therefore, two values of $\partial \mathrm{p} / \partial \mathrm{t}$ can be calculated by the equation (5), i.e., -0.027 and -0.042 for both cases of $\mathrm{RC}$ shorter and longer than 50 msec. respectively. Substituting these two values of constants for the equation $\left(5^{\prime}\right)$, the $\lambda-\log$ I curves obtained are shown by thick curves in Fig. 2 (A) and (B), respectively. The $r$ curves were obtained by using Steinhardt's data ${ }^{2)}$ for red light (test field of $20^{\circ}$ ), and the $w$ curves by using his data for white light (test field of $20^{\circ}$ ). As can be seen in Fig. 2 (A) and (B), the $r$ and $w$ curves are approximately corresponding to the respective thin curves for red and green, which were obtained actually in this experiment, although the $r$ and $w$ curves obtained from the Steinhardt's data covered only a narrow range of radiance. Thus, this consistent results of the experimental data with the formula $\left(5^{\prime}\right)$, indicate that $\partial \mathrm{p} / \partial \mathrm{t}$ can be expressed approximately by two constants corresponding to the time-constants shorter or longer than $50 \mathrm{msec}$, as mentioned above. Integrating the equation $\partial \mathrm{p} / \partial \mathrm{t}=$ constant, we obtain

$$
\begin{array}{ll}
\mathrm{p}=-0.027 \mathrm{t}+\mathrm{C}_{1} & \text { for } \mathrm{t}<50 \mathrm{msec} . \\
\mathrm{p}=-0.042 \mathrm{t}+\mathrm{C}_{2} & \text { for } \mathrm{t}>50 \mathrm{msec} .
\end{array}
$$

respectively, where $\mathrm{C}_{1}$ and $\mathrm{C}_{2}$ are constants of integration. However, when we put 


$$
\begin{array}{ll}
p=9.3 \mathrm{e}^{-\mathrm{t} / 285}-5.8 & \text { for } \mathrm{t}<50 \mathrm{msec}, \\
\mathrm{p}=-0.042 \mathrm{t}+5.2 & \text { for } \mathrm{t}>50 \mathrm{msec},
\end{array}
$$

respectively, far better agreement of these formulae with the actual data of the $\mathrm{p}-\mathrm{t}$ relation can be obtained.

As discussed above, it is noteworthy that the correlation among the electric threshold $(\mathrm{V})$, brightness discrimination and the time-constant (RC) of the stimulating current was elucidated by expressing in an experimental formula, and that the different values of parameter $p$ in the formula relate closely to the site of electrostimulation in the human retina. Another deduction worth noting is that the accommodation constant of the retina is inversely proportional to the Weber fraction in brightness discrimination.

\section{SUMmary}

Using, as an index of excitation, the electrical phosphene elicited by the exponentially increasing current running through the eyeballs, the relation of the electric threshold and accommodation in the human retina to brightness discrimination was studied systematically with the eyes adapted to monochromatic rays of varying wavelengths.

1. All of the accommodation curves obtained were of duplex nature, being divided into two sections by an inflexion at about $50-80 \mathrm{msec}$. in RC.

2. Hill's $\lambda$-log-radiance (I) curves were obtained separately for both cases of the time-constants (RC) shorter and longer than $50 \mathrm{msec}$. Both families of the curves for both cases of $\mathrm{RC}$, were different not only in shape but also in arrangement order of the curves depending on the wavelength of the adapting light.

3. Hill's $\lambda$ has a parallel relation to spectral luminosity of the adapting light, when the adaptng colored light was of equal energy.

4. It was found that the electric threshold (V) can be expressed by a simple formula as a function of the Weber fraction (W) in brightness discrimination and the intensity (I) of the adapting light, as follows,

$$
\mathrm{V}=\mathrm{A}\left(\log \mathrm{W}^{\mathrm{p}}+\log \mathrm{I}\right)+\mathrm{B} \text {, }
$$

where $A$ and $B$ are constants and $\mathrm{p}$ is the parameter which relates to the time-constant of the stimulating current.

5. The relation between the accommodation constant (Hill's $\lambda$ ) and the intensity of the adapting light, was deduced mathematically from the formula stated above, and it was indicated that the Hill's $\lambda$ is inversely proportional to the Weber fraction in brightness discrimination.

I wish to express my cordial thanks to Prof. T. Mita who has given me 
valuable advice throughout the experiments and many constructive criticisms in preparing this paper, and also to Dr. A. Akihama for his helpful assistance throughout this work.

\section{References}

1) Sato, M., Jap. J. Physiol., 1958, 8, 1.

2) Steinhardt, S., J. Gen. Physiol., 1936, 20, 185.

3) Blackwell, H. R., J. Opt. Soc. Amer., 1946, 36, 624.

4) Hill, A. V., Proc. Roy. Soc., 1936, 119, 305.

5) Sato., M, Suzuki, T. \& Akihama, A., Jap. J. Physiol., 1957, 7, 29.

6) Yaegashi, S., Tohoku J. Exp. Med., 1958, 67, 163.

7) Mita, T., Fujimaki, E. \& Takahashi, R., Jap. J. Physiol., 1954, 4, 115.

8) Mita, T., Fujimaki, E. \& Yaegashi, S., Tohoku J. Exp. Med., 1956, 65, 45.

9) Motokawa, K. \& Iwama, K., Tohoku J. Exp. Med., 1949, 50, 25. 